
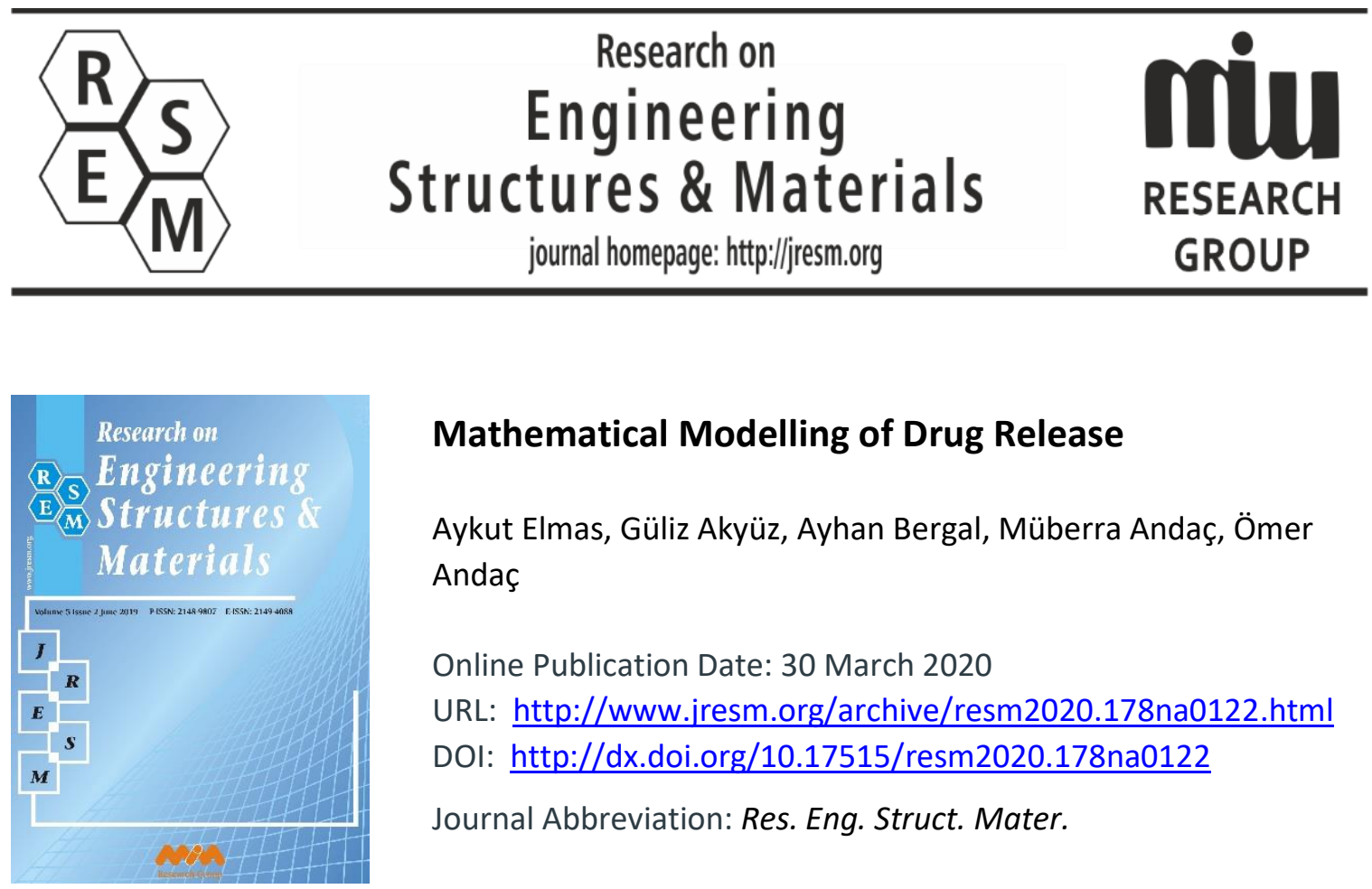

\title{
Mathematical Modelling of Drug Release
}

Aykut Elmas, Güliz Akyüz, Ayhan Bergal, Müberra Andaç, Ömer Andaç

Online Publication Date: 30 March 2020

URL: http://www.jresm.org/archive/resm2020.178na0122.html

DOI: http://dx.doi.org/10.17515/resm2020.178na0122

Journal Abbreviation: Res. Eng. Struct. Mater.

\section{To cite this article}

Elmas A, Akyuz G, Bergal A, Andac M, Andac O. Mathematical Modelling of Drug Release. Res. Eng. Struct. Mater., 2020; 6(4): 327-350.

\section{Disclaimer}

All the opinions and statements expressed in the papers are on the responsibility of author(s) and are not to be regarded as those of the journal of Research on Engineering Structures and Materials (RESM) organization or related parties. The publishers make no warranty, explicit or implied, or make any representation with respect to the contents of any article will be complete or accurate or up to date. The accuracy of any instructions, equations, or other information should be independently verified. The publisher and related parties shall not be liable for any loss, actions, claims, proceedings, demand or costs or damages whatsoever or howsoever caused arising directly or indirectly in connection with use of the information given in the journal or related means.

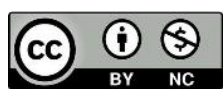
Published articles are freely available to users under the terms of Creative Commons Attribution - NonCommercial 4.0 International Public License, as currently displayed at here (the "CC BY - NC"). 


\title{
Research on Engineering Structures \& Materials
}

journal homepage: http://jresm.org

Review Article

\section{Mathematical modelling of drug release}

\author{
Aykut Elmas*1,a, Güliz Akyüz ${ }^{1, b}$, Ayhan Bergal1,c, Müberra Andaç̧,2,d , Ömer Andaç̧,2,e \\ ${ }^{1}$ Department of Nanoscience and Nanotechnology, Ondokuz Mayis University, Samsun, Turkey \\ ${ }^{2}$ Department of Chemistry, Ondokuz Mayis University, Samsun, Turkey
}

\begin{tabular}{ll}
\hline Article Info & Abstract \\
\hline $\begin{array}{l}\text { Article history: } \\
\text { Received 22 Jan } 2020 \\
\text { Revised 19 Mar 2020 }\end{array}$ & $\begin{array}{l}\text { Mathematical models of the drug release have been used in the drug delivery } \\
\text { (DD) field for more than } 50 \text { years by the scientists in the drug development } \\
\text { process. These models not only help scientists to learn the dynamics of the drugs } \\
\text { release, but also help them to save money and time by helping to design more } \\
\text { effective experiments. There is no model in the literature that covers all drug } \\
\text { release scenarios. Also, some system-specific models have complex } \\
\text { mathematical equations and these models are not suitable for general use. Zero }\end{array}$ \\
Keywords: & $\begin{array}{l}\text { Order Model, First Order Model, Higuchi Model, Peppas Model and Hixon Crowell } \\
\text { Model are used in 85\% of drug release studies in total. The popularity of these } \\
\text { models comes from their simplicity, easy mathematical expressions and } \\
\text { implementation. In this review, mathematical derivations of these five models } \\
\text { are shown in detail. The points to be considered during the derivation and the } \\
\text { problems that may be encountered are carefully explained along with their } \\
\text { solutions. In addition, the application of the models to drug release data and the } \\
\text { points to be considered were obtained by writing from the scratch without using } \\
\text { any ready software while obtaining the fit function. In this way, many problems } \\
\text { are better understood, and their solutions are explained. Finally, the obtained fit } \\
\text { functions are interpreted. }\end{array}$ \\
Mathematical & $\begin{array}{l}\text { Modelling; } \\
\text { Controlled Release }\end{array}$
\end{tabular}

(C) 2020 MIM Research Group. All rights reserved.

\section{Introduction}

In the field of DD, deriving a mathematical model has been a challenging subject for scientists who want to predict drug release. A strong mathematical model can reduce the number of required experiments thanks to its prediction ability. And, these estimates prevent waste of time and money by helping to prepare more efficient experiments.

Up to date, there have been many attempts to describe drug release mathematically [1-4]. However, these models either failed to cover the entire DD area, or designed for very rare special systems and were not suitable for general use. Therefore, they cannot give accurate results, and some of them are too complex to apply to release data. In 1961, legendary professor Takeru Higuchi published his famous work on mathematical modelling of drug delivery [5]. And his equation becomes famous because it was theoretically stable, easy to apply, and has wide range of use which makes him called as a "father" of mathematical modelling of drug delivery. His surprisingly simple description of drug release from an ointment base was the beginning of the quantitative treatment of drug release. After Higuchi, there has been modifications of his model for describing porous systems and different geometries [1].

\footnotetext{
*Corresponding author: aykutdiamond@gmail.com

a orcid.org/0000-0002-7721-4088; borcid.org/0000-0002-3522-9716; corcid.org/0000-0002-5930-9104;

dorcid.org/0000-0001-7262-9762; e orcid.org/0000-0003-3641-9690

DOI: http://dx.doi.org/10.17515/resm2020.178na0122
}

Res. Eng. Struct. Mat. Vol. 6 Iss. 4 (2020) 327-350 
A mathematical framework has been developed for studying to drug release from hydroxypropyl methyl cellulose (HPMC) tablets [6, 7]. A comprehensive model has been developed to describe dissolution and swelling behaviors from cylindrical HPMC tablets [8-10]. And an extension of this study has been developed for different coordinates [1113]. This model was the most advanced one for the polymers undergoing erosion and swelling. For dissolving polymer system, first model has been developed in 1988 [14] and modified for macromolecular movements [15]. To overcome the blood-brain barrier, a finite element model was developed for central nervous system in 1997 [16]. Then, doxorubicin supported models developed for breast cancer [17]. Most of these models are based on the Fick's diffusion equation [18] and solutions for different variations [19]. Unfortunately, none of these models yield perfect solution. In drug delivery studies, there is no model covers all the problems. When developing a model, the following issues should be carefully considered.

- Only dominant features should be added to the model. Otherwise the equation would be too complex.

- Theory should be supported by the experiments.

- There is no mathematical theory that can be applied to all DD systems.

- Theory should be abandoned when it fails, even if it is compatible with the experiment.

The purpose of this article is to provide information about popular mathematical models. Since it is rarely encountered in the field of DD, the mathematics in deriving models is clearly explained and possible problems are shown with their solutions. Finally, it is shown that fitting procedure of the models into a release data. In the meantime, a program was written from scratch and the places that need attention are highlighted without using existing software. According to the work of Caccavo, some models are dominating the DD field [20]. They are, Zero Order model 18.9\%, First Order model 15.6\%, Higuchi model $19.4 \%$, Peppas model $30 \%$ and Hixon-Crowell model $7.8 \%$ of usage frequency. It is also focused on these models in this work.

\section{Derivation of the Models}

For models that require long calculation, intermediate steps have been neglected. This neglect could mislead scientists because there may be some important points that guide calculations. Also jumping directly to the model result, makes harder to understand the theoretical concept of the model derivations.

The drug begins to dissolve in a special solvent such as water, blood, or gastric liquid. However, the dissolved molecules are not immediately dispersed into all the solvent called "bulk liquid". An imaginary layer occurs on the surface of the drug called "stagnant liquid". In sink conditions, concentration of the drug molecules is always considered zero for the simplification of the diffusion calculations. Because simplification assumes constant diffusion and gradient. The drug molecules will diffuse from higher concentration to the lesser concentration through stagnant liquid by the lead of Fick's law of diffusion [18].

$$
J=-D_{f} \frac{d c}{d x}
$$


Where $J$ is the amount of substance passing perpendicularly through a unit surface area per time known as "flux". $D_{f}$ is the diffusion coefficient and $d c / d x$ is the concentration gradient. The reason for the negative sign is, the gradient operator has the negative slope while concentration decreases with position. But the flux is a direction independent absolute quantity. Which means, there is flux or there is no flux. So, there shouldn't be a negative flux, that's why negative sign is added in the equation.

\subsection{Zero Order Model}

Zero Order model based on the reaction kinetics of the chemistry. According to these kinetics, speed of the reaction can be described as,

$$
R=K_{0}[A]^{0}
$$

Where $R$ is the reaction speed, $[A]^{0}$ represents the concentration of $A$ which entering the raction with zero degree, and $K_{0}$ is the zero order rate constant. Also, reaction speed can be written as,

$$
R=-\frac{\Delta[A]}{\Delta t}
$$

Where $t$ is time. If reaction speed can be represented with two different equations, one can substitute equations (2) and (3).

$$
\frac{d[A]}{d t}=-K_{0}
$$

Or according to experimental conditions, one can write $C$ in stead of $[A]$ to represent the concentration.

$$
\frac{d C}{d t}=-K_{0}
$$

Then, applying simple mathematics,

$$
\begin{aligned}
& d C=-K_{0} d t \rightarrow \int_{C_{0}}^{C_{t}} d C=-K_{0} \int d t \\
& \left.(C)\right|_{C_{0}} ^{C_{t}}=-K_{0} t \rightarrow C_{t}-C_{0}=-K_{0} t \\
& C_{t}=C_{0}-K_{0} t
\end{aligned}
$$


Here in eqn. (4) we have a linear representation of drug release as a Zero Order model. Advantages and disadvantages of this linearity will be discussed in experimental analysis part.

\subsection{First Order Model}

For the derivation of the First Order model, mathematical theory is same as the Zero Order model. Eqn. (3) remains same but eqn. (2) becomes first degree of concentration of substance $A$ which enters the reaction.

$$
R=K_{1}[A]^{1}
$$

Same as Zero Order model, one can substitute eqns. (3) and (5).

$$
\begin{aligned}
& \frac{d C}{d t}=-K_{1} C \quad \rightarrow \quad \frac{d C}{C}=-K_{1} d t \\
& \int_{C_{0}}^{C} \frac{1}{C} d C=-\left.K_{1} \int d t \rightarrow \ln (C)\right|_{C_{0}} ^{C}=-K_{1} t \\
& \ln (C)-\ln \left(C_{0}\right)=-K_{1} t \\
& \ln \left(\frac{C}{C_{0}}\right)=-K_{1} t
\end{aligned}
$$

Here one should remember the rule which is $\log (x)=\ln (x) / \ln (10)$. So,

$$
\ln (10) \log (x)=\ln (x)
$$

By, substituting eqns. (6) and (7) one can get,

$$
\begin{aligned}
& \ln (10) \log \left(\frac{C}{C_{0}}\right)=-K_{1} t \quad \rightarrow \quad \ln (10)=2.303 \\
& \log \left(\frac{C}{C_{0}}\right)=\frac{-K_{1} t}{2.303} \rightarrow \log (C)-\log \left(C_{0}\right)=\frac{-K_{1} t}{2.303} \\
& \log (C)=\log \left(C_{0}\right)-\frac{-K_{1} t}{2.303}
\end{aligned}
$$


Eqn. (8) can be considered the final representation of First Order model. This model also shows similarities with Zero Order model by linearity but thanks to logarithmic representation of the equation, there should be some important point to focus on while fitting procedure, which will be discussed in experimental analysis chapter.

\subsection{Higuchi Model}

Fifty years ago professor Takeru Higuchi created his famous equation that allows prediction of drug release from thin ointment films [5]. Before his derivation, he made some assumptions to overcome same problems. These assumptions are should be taken into account otherwise it might cause to misuse of the model.

- Transfer of the drug inside the ointment is slow, but inside the skin is fast. In these conditions one could observe stable diffusion.

- Skin acts like a perfect sink, which means the concentration of the drug is ignored. So, it is assumed the concentration in the skin is never increase.

- Concentration in the film is much more than the solubility of the drug at the ointment base.

- Drug separated to the ointment base uniformly and size of the drug particles much less than the ointment layer thickness.

- $\quad$ Speed of the drug solubility in the ointment base faster than the diffusion speed of the dissolved drug particles there. This assumption is important because diffused particle is immediately replaced by the new one.

- Diffusion coefficient in the ointment base is stable and not dependent neither time nor position of the layer.

- Edge effects are ignored. Skin surface area is much bigger than the thickness of the ointment film, so one can say the diffusion occurs in one dimension.

- Ointment base neither dissolves nor swells.

In the frame of these assumptions Higuchi made his derivations from graphic below (Figure 1). 


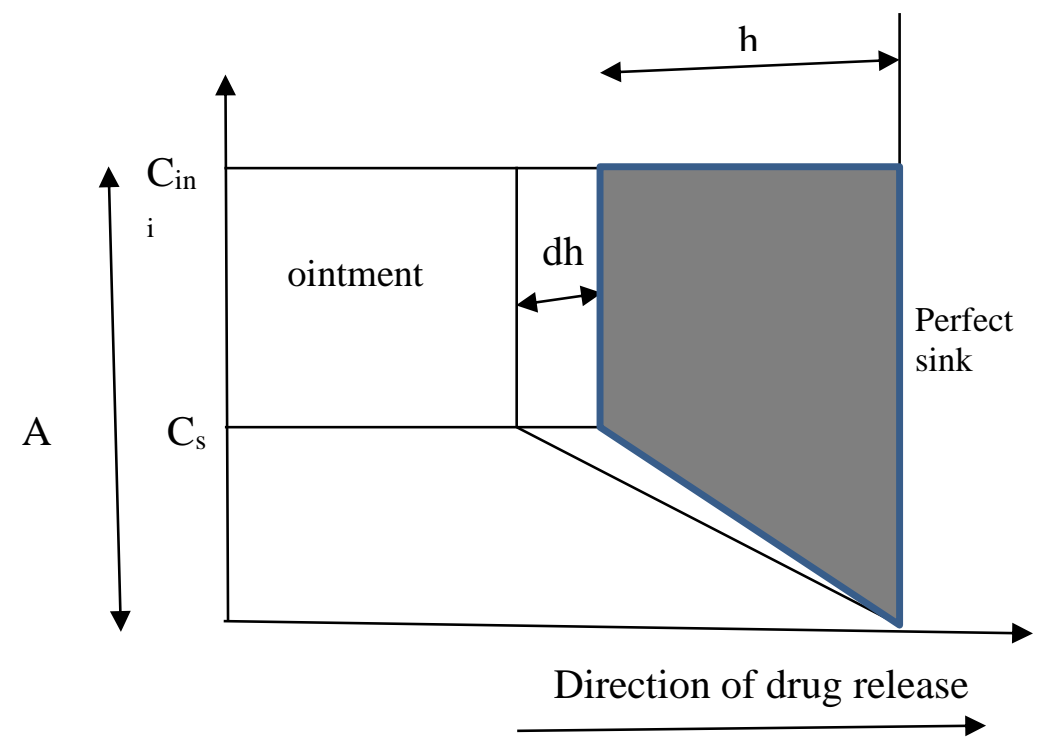

Fig. 1 Drug diffusion from ointment, where $\mathrm{C}_{\text {ini }}$ is the initial drug concentration, $\mathrm{C}_{\mathrm{s}}$ is the drug solubility, horizontal axis is the diffusion direction of the molecules and the vertical axis is the drug concentration.

Here we can think that the perfect sink is the human skin and the grey area is soluted drugs membrane. If it is donated $Q$ is the amount of absorbed at time $t$ per unit area of exposure, and $A$ is the concentration of drug expressed in units $/ \mathrm{cm}^{3}$, surface area of the grey trapezoid in Figure 1 (right hand side of the eqn. (9)) corresponds to $Q$.

$$
Q=h\left(A-\frac{C_{s}}{2}\right)
$$

Here, $h$ is an unknown parameter which should be fund. Rearranging Eqn. (9)

$$
\begin{aligned}
& Q=h\left(A-\frac{C_{s}}{2}\right) \rightarrow Q=A h-\frac{C_{s}}{2} h \\
& d Q=A d h-\frac{1}{2} C_{s} d h
\end{aligned}
$$

Also professor Higuchi foresaw that Fick's law [18] can be written as,

$$
\frac{d Q}{d t}=D C_{s} / h \quad \rightarrow \quad d Q=D \frac{C_{s}}{h} d t
$$

Where $D$ is the diffusion constant. Substituting eqns. (10) and (11) we can get, 


$$
A d h-\frac{1}{2} C_{s} d h=D \frac{C_{s}}{h} d t
$$

Dividing both sides by $d t$ we get,

$$
\begin{aligned}
& A \frac{d h}{d t}-\frac{1}{2} C_{s} \frac{d h}{d t}=\frac{D C_{s}}{h} \frac{d t}{d t} \quad \rightarrow \quad A \frac{d h}{d t}-\frac{1}{2} C_{s} \frac{d h}{d t}=\frac{D C_{s}}{h} \\
& \frac{d h}{d t}\left(A-\frac{1}{2} C_{s}\right)=\frac{D C_{s}}{h} \rightarrow \frac{d h}{d t}\left(A-\frac{1}{2} C_{s}\right) h=D C_{s} \\
& d h\left(A-\frac{1}{2} C_{s}\right) h=D C_{s} d t \quad \rightarrow \quad \frac{\left(A-\frac{1}{2} C_{s}\right)}{D C_{s}} h d h=d t
\end{aligned}
$$

Both multiplying and dividing by 2 will simplify the calculations,

$$
\begin{aligned}
& \frac{2}{2} \frac{\left(A-\frac{1}{2} C_{s}\right)}{D C_{s}} h d h=d t \rightarrow \frac{\left(2 A-C_{s}\right)}{2 D C_{s}} h d h=d t \\
& \frac{\left(2 A-C_{s}\right)}{2 D C_{s}} \int h d h=\int d t \rightarrow \frac{\left(2 A-C_{s}\right)}{2 D C_{s}}\left(\frac{h^{2}}{2}\right)=t \\
& \frac{\left(2 A-C_{s}\right)}{4 D C_{s}} h^{2}=t \quad \rightarrow \quad h^{2}=\frac{4 D C_{s} t}{2 A-C_{s}} \\
& h=2 \sqrt{\frac{D C_{s} t}{2 A-C_{s}}}
\end{aligned}
$$

Now, $h$ can be represented by eqn (12). Substituting into Eqn. (9),

$$
\begin{aligned}
& Q=A h-\frac{C_{s}}{2} h \rightarrow Q=2 A \sqrt{\frac{D C_{s} t}{2 A-C_{s}}}-\frac{C_{s}}{2} 2 \sqrt{\frac{D C_{s} t}{2 A-C_{s}}} \\
& Q=\sqrt{\frac{D C_{s} t}{2 A-C_{s}}}\left(2 A-C_{s}\right) \rightarrow Q=\sqrt{\frac{D C_{s} t\left(2 A-C_{s}\right)^{2}}{\left(2 A-C_{s}\right)}} \\
& Q=\sqrt{D C_{s} t\left(2 A-C_{s}\right)}
\end{aligned}
$$

According to Higuchi, if $C_{s} \ll A$, then $\left(2 A-C_{S}\right) \approx 2 A$. So $Q$ becomes 


$$
Q=\sqrt{2 A D C_{s} t}
$$

Here $A, D$ and $C_{s}$ are constant. If they merge into one sinle constant like $k$ final equation becomes popular Higuchi model form as,

$$
Q=k \sqrt{t}
$$

As it seen from the Eqn. (13) Higuchi model is very simple equation. Zero Order and First Order models were linear representations of the drug release but, Higuchi model defines the release with square root of time which makes sense and strong relationship with the experiments.

\subsection{Peppas Model}

Peppas model also based on diffusion mechanics[19-21]. For the starting point, one may think the diffusion occurs in one dimension which is not real but simplifies the calculations. Figure 2

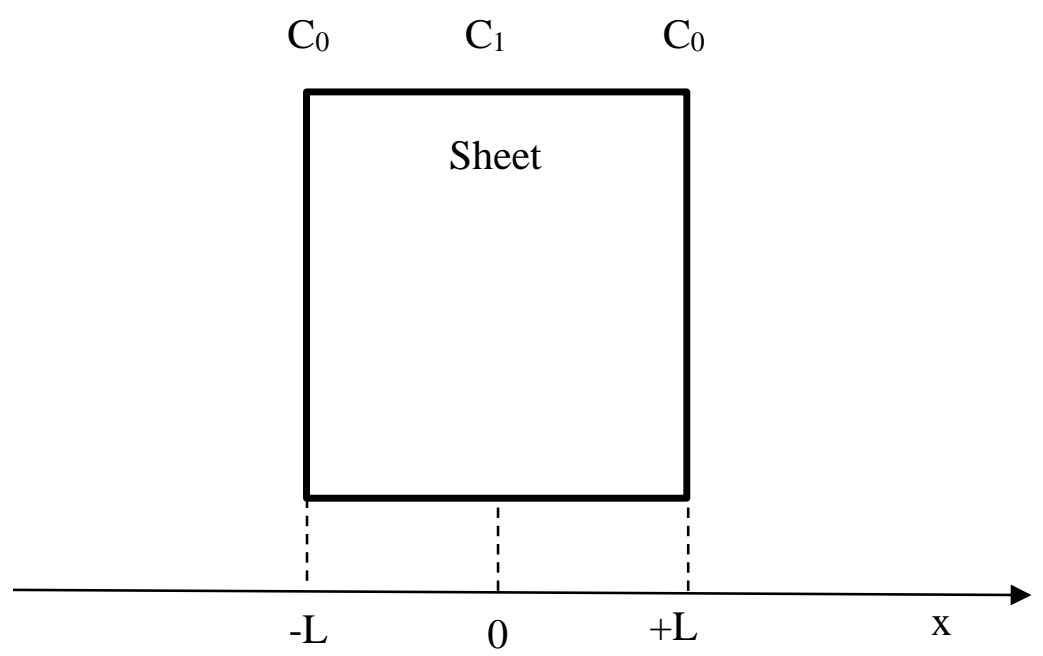

Fig. 2 Diffusion from a plane sheet. C, L and x represents concentration, length and direction respectively.

Defining boundary conditions as,

$$
t=0 \quad, \quad-L<x<L \quad, \quad C=C_{\text {in }}
$$




$$
\begin{aligned}
& t>0, \quad x \mp L, \quad C=C_{0} \\
& t \geq 0, \quad \frac{d C}{d x}=0, \quad x=0
\end{aligned}
$$

Where $C_{\text {in }}$ represents the initial concentration and,

$$
C_{0}=C_{\text {surface }}-C_{\text {ext }}
$$

Where $C_{0}$ is the concentration at $\mp L, C_{\text {surface }}$ is the surface contentration of the plane sheet and $C_{\text {ext }}$ is the external concentration. After defining boundary conditions, one may focus on the Fick's second diffusion equation [18].

$$
\frac{d C}{d t}=D \frac{d^{2} C}{d x^{2}}
$$

Where $D$ is the diffusion constant. In the Eqn. (18) concentration is both function of time and position. This differential equation is called partial differential equation and could be solved by separation of variables technique as,

$$
C(x, t)=C_{x} C_{t}
$$

If it is substituted Eqn. (19) into Eqn. (18),

$$
\frac{d C_{t}}{d t} C_{x}=D C_{t} \frac{d^{2} C_{x}}{d x^{2}}
$$

Rearranged as,

$$
\underbrace{\frac{1}{C_{t}} \frac{d C_{t}}{d t}}_{\text {only depends on } t}=\underbrace{\frac{D}{C_{x}} \frac{d^{2} C_{x}}{d x^{2}}}_{\text {only depends on } x}
$$

Here in Eqn. (20) two different differential equations depending different variables equal each other. So, both equations should be equal same constant. That constant could be chosen anything. In this part it is chosen $-\lambda^{2} D$ for the convenience of the further calculations. By focusing left hand side of the Eqn. (20)

$$
\frac{1}{C_{t}} \frac{d C_{t}}{d t}=-\lambda^{2} D
$$

We get the first ordinary differential equation. And the right hand side of the Eqn. (20) 


$$
\frac{D}{C_{x}} \frac{d^{2} C_{x}}{d x^{2}}=-\lambda^{2} D
$$

Becomes the second ordinary differential equation. Solutions of the Eqns. (21) and (22) are known well by the calculus. These solutions are,

$$
\begin{aligned}
& C_{t}=\text { Constant } \times \operatorname{Exp}\left(-\lambda^{2} D t\right) \\
& C_{x}=A \times \operatorname{Sin}(\lambda \mathrm{x})+\mathrm{B} \times \operatorname{Cos}(\lambda \mathrm{x})
\end{aligned}
$$

Respectively. So the Eqn. (19) should be the multiplication of Eqns. (23) and (24).

$$
C(x, t)=C_{x} C_{t}=\text { Constant } \times(A \operatorname{Sin}(\lambda x)+B \operatorname{Cos}(\lambda x)) \operatorname{Exp}\left(-\lambda^{2} D t\right)
$$

For all states, general solution of Eqn. (25),

$$
C=\sum_{n=0}^{\infty}\left(A_{n} \operatorname{Sin}\left(\lambda_{n} x\right)+B_{n} \operatorname{Cos}\left(\lambda_{n} x\right)\right) \operatorname{Exp}\left(-\lambda_{n}^{2} D t\right)
$$

Eqn. (26) will be the general solution but there are three unknown parameters like $A_{n}, B_{n}$ and $\lambda_{n}$. To find these parameters one should apply the boundary conditions. But first Eqn. (17) should be understood well. From the Figure 2 if $C_{\text {surface }}$ and $C_{\text {external }}$ are equal, $C_{0}=$ $C_{\text {surf. }}-C_{\text {ext. }}$. becomes zero. That is why there is no obstacle to write concentration like $C-$ $C_{0}$ and $C_{0}$ is constant.

$$
C-C_{0}=\sum_{n=0}^{\infty}\left(A_{n} \operatorname{Sin}\left(\lambda_{n} x\right)+B_{n} \operatorname{Cos}\left(\lambda_{n} x\right)\right) \operatorname{Exp}\left(-\lambda_{n}^{2} D t\right)
$$

Now, let's focus on the boundary conditions. According to Eqn. (16) while $\geq 0, d C / d x=0$ , at $x=0$ mid plane. Taking derivative of Eqn. (27) according to $x$, at $x=0$ one get,

$$
\begin{aligned}
& \frac{d C}{d x}=0=\sum_{n=0}^{\infty} \underbrace{\lambda_{n}}_{\text {constant }}[\underbrace{A_{n} \operatorname{Cos}\left(\lambda_{n} 0\right)+B_{n} \operatorname{Sin}\left(\lambda_{n} 0\right)}_{=0}] \underbrace{\operatorname{Exp}\left[-\lambda_{n}^{2} D t\right]}_{\neq 0, t \geq 0} \\
& \underbrace{A_{n}}_{=0} \underbrace{\operatorname{Cos}(0)}_{=1}+\underbrace{B_{n}}_{\neq 0} \underbrace{\operatorname{Sin}(0)}_{=0}=0 \rightarrow A_{n}=0, B_{n} \neq 0 \\
& C-C_{0}=\sum_{n=0}^{\infty}\left[B_{n} \operatorname{Cos}\left(\lambda_{n} x\right)\right] \operatorname{Exp}\left[-\lambda_{n}^{2} D t\right]
\end{aligned}
$$


The parameter $A_{n}$ has been known and $B_{n}$ is not equal zero. To keep searching the parameters lets apply another boundary condition form Eqn. (15) as, $>0, x=\mp L, C=$ $C_{0} \rightarrow C-C_{0}=0$.

$$
\begin{aligned}
& \text { at } x=L \rightarrow C-C_{0}=0=\sum_{n=0}^{\infty}[\underbrace{B_{n} \operatorname{Cos}\left(\lambda_{n} L\right)}_{=0}] \underbrace{\operatorname{Exp}\left[-\lambda_{n}^{2} D t\right]}_{\neq 0, t>0} \\
& \underbrace{B_{n}}_{\neq 0} \underbrace{\operatorname{Cos}\left(\lambda_{n} L\right)}_{=0}=0 \rightarrow \operatorname{Cos}\left(\lambda_{n} L\right)=0 \rightarrow \lambda_{n} L=(2 n+1) \frac{\pi}{2} \\
& \lambda_{n}=\frac{(2 n+1) \pi}{2 L}
\end{aligned}
$$

By substituting Eqn. (30) into Eqn. (29) we get,

$$
C-C_{0}=\sum_{n=0}^{\infty}\left[B_{n} \operatorname{Cos}\left(\frac{(2 n+1) \pi x}{2 L}\right)\right] \operatorname{Exp}\left[-\frac{(2 n+1)^{2} \pi^{2}}{4 L^{2}} t D\right]
$$

With Eqn. (31) there is only one parameter remains unknown which is $B_{n}$. To find that parameter the first boundary condition could be applied in Eqn. (14). After applying the first boundary condition, exponential part of the Eqn. (31) equals 1 . Considering the concentration as, $C=C_{1}$ we get,

$$
C_{1}-C_{0}=\sum_{n=0}^{\infty} B_{n} \operatorname{Cos}\left(\frac{(2 n+1) \pi x}{2 L}\right)
$$

To solve the Eqn. (32) superposition theorem should be applied. Both sides of the equation should be multiplied by $\operatorname{Cos}((2 P+1) \pi x / 2 L)$ and, should be integrated from $-L$ to $L . P$ is an arbitrary parameter which will be eliminated in further steps of the calculations.

$$
\begin{aligned}
\left(C_{1}-C_{0}\right) \int_{-L}^{L} \operatorname{Cos}\left(\frac{(2 P+1) \pi x}{2 L}\right) d x \\
\quad=\sum_{n=0}^{\infty} B_{n} \int_{-L}^{L} \operatorname{Cos}\left(\frac{(2 P+1) \pi x}{2 L}\right) \operatorname{Cos}\left(\frac{(2 n+1) \pi x}{2 L}\right) d x
\end{aligned}
$$

According to superposition theorem,

$$
\int_{-L}^{L} \operatorname{Cos}\left(\frac{(2 P+1) \pi x}{2 L}\right) \operatorname{Cos}\left(\frac{(2 n+1) \pi x}{2 L}\right) d x= \begin{cases}0, & P \neq n \\ L & , \quad P=n\end{cases}
$$

Equality of zero will not be physical because it is known that there is a concentration of substance. So, it is chosen $P=n$. 


$$
\left(C_{1}-C_{0}\right) \int_{-L}^{L} \operatorname{Cos}\left(\frac{(2 n+1) \pi x}{2 L}\right) d x=\sum_{n=0}^{\infty} B_{n} L
$$

To solve the Eqn. (33), change of variables technique is applied as,

$$
\begin{aligned}
& U=\frac{(2 n+1) \pi x}{2 L}, \quad d U=\frac{(2 n+1) \pi x}{2 L} d x, \quad d x=\frac{2 L}{(2 n+1) \pi} d U \\
& x=-L \quad \rightarrow \quad U=-\frac{(2 n+1) \pi}{2}, \quad x=L \quad \rightarrow \quad U=\frac{(2 n+2) \pi}{2}
\end{aligned}
$$

Then the Eqn. (33) becomes,

$$
\left(C_{1}-C_{0}\right) \times \int_{-\frac{(2 n+1) \pi}{2}}^{\frac{(2 n+1) \pi}{2}} \operatorname{Cos}(U) \times \frac{2 L}{(2 n+1) \pi} d U=\sum_{n=0}^{\infty} B_{n} L
$$

Rearranging,

$$
\begin{aligned}
& \left(C_{1}-C_{0}\right) \times \frac{2 L}{(2 n+1) \pi} \int_{-\frac{(2 n+1) \pi}{2}}^{\frac{(2 n+1) \pi}{2}} \operatorname{Cos}(U) d U=\sum_{n=0}^{\infty} B_{n} L \\
& \left(C_{1}-C_{0}\right) \times \frac{2 L}{(2 n+1) \pi}(\sin (U)) \mid \begin{array}{l}
\frac{(2 n+1) \pi}{2} \\
-\frac{(2 n+1) \pi}{2}
\end{array}=\sum_{n=0}^{\infty} B_{n} L \\
& \left(C_{1}-C_{0}\right) \times \frac{4 L}{(2 n+1) \pi} \operatorname{Sin}\left(\frac{(2 n+1) \pi}{2}\right)=\sum_{n=0}^{\infty} B_{n} L
\end{aligned}
$$

To simplify the Eqn. (34) it is observed the behavior of the trigonometric part by assigning numbers for $n$.

$$
\left.\begin{array}{c}
n=0 \rightarrow \sin \left(\frac{(2 n+1) \pi}{2}\right)=\sin \left(\frac{\pi}{2}\right)=1 \\
n=1 \rightarrow \sin \left(\frac{(\pi \pi}{2}\right)=-1 \\
n=2 \rightarrow 1 \\
n=3 \rightarrow-1
\end{array}\right\}=\begin{array}{ccc}
1 \quad \text { if } & n=\text { even } \\
-1 \quad \text { if } & n=\text { odd }
\end{array}
$$

Then,

$$
\operatorname{Sin}\left(\frac{(2 n+1) \pi}{2}\right)=(-1)^{n}
$$

Substituting Eqn. (35) into Eqn. (34) and canceling $L$ 's from both side, $B_{n}$ can be found as, 


$$
B_{n}=\frac{\left(C_{1}-C_{0}\right) 4(-1)^{n}}{(2 n+1) \pi}
$$

Substituting Eqn. (36) into Eqn. (31),

$$
C-C_{0}=\sum_{n=0}^{\infty} \frac{\left(C_{1}-C_{0}\right) 4(-1)^{n}}{(2 n+1) \pi} \operatorname{Cos}\left(\frac{(2 n+1) \pi x}{2 L}\right) \operatorname{Exp}\left[-\frac{(2 n+1)^{2} \pi^{2}}{4 L^{2}} t D\right]
$$

At time $t$ amount of substance which is transferring inside or ourside the sheet is $M_{t}$ can be found by the integration of the flux $\left(f l u x=D\left|\frac{d c}{d x}\right|\right)$ at the surface.

$$
M_{t}=\int_{0}^{t} D\left|\frac{d C}{d x}\right|_{x=-L} d t+\int_{0}^{t} D\left|\frac{d C}{d x}\right|_{x=-L} d t=2 \int_{0}^{t} D\left|\frac{d C}{d x}\right|_{x=\mp L} d t
$$

Concentration $C$ is known by Eqn. (37), $M_{t}$ will be searched.

$$
\begin{aligned}
& \left|\frac{d C}{d x}\right|_{\infty}^{\infty} \frac{\left(C_{1}-C_{0}\right) 4(-1)^{n}}{(2 n+1) \pi}\left|\frac{(-1)(2 n+1) \pi}{2 L} \operatorname{Sin}\left(\frac{(2 n+1) \pi x}{2 L}\right)\right| \operatorname{Exp}\left[-\frac{(2 n+1)^{2} \pi^{2}}{4 L^{2}} t D\right] \\
& \left|\frac{d C}{d x}\right|_{x=\mp L}=\frac{2\left(C_{1}-C_{0}\right)}{L} \sum_{n=0}^{\infty}(-1)^{n} \underbrace{\operatorname{Sin}\left(\frac{(2 n+1) \pi x}{2 L}\right)}_{x=\mp L \rightarrow(-1)^{n}} \operatorname{Exp}\left[-\frac{(2 n+1)^{2} \pi^{2}}{4 L^{2}} t D\right] \\
& M_{t}=2 D \int_{0}^{t}\left|\frac{d C}{d x}\right|_{x=\mp L} d t \\
& M_{t}=\frac{4 D\left(C_{1}-C_{0}\right)}{L} \int_{0}^{t} \sum_{n=0}^{\infty} \operatorname{Exp}\left[-\frac{(2 n+1)^{2} \pi^{2} D t}{4 L^{2}}\right] d t
\end{aligned}
$$

Integration in Eqn. (38) can be solved by change of variables technique as,

$$
\begin{aligned}
& U=-\frac{(2 n+1)^{2} \pi^{2} D t}{4 L^{2}}, d U=-\frac{(2 n+1)^{2} \pi^{2} D}{4 L^{2}} d t, d t=-\frac{4 L^{2}}{(2 n+1)^{2} \pi^{2} D} \\
& t=0 \quad \rightarrow \quad U=-\frac{(2 n+1)^{2} \pi^{2} D 0}{4 L^{2}}=0
\end{aligned}
$$




$$
t=t \quad \rightarrow \quad U=-\frac{(2 n+1)^{2} \pi^{2} D t}{4 L^{2}}
$$

Substituting into Eqn. (38)

$$
M_{t}=\frac{4 D\left(C_{1}-C_{0}\right)}{L} \int_{0}^{-\frac{(2 n+1)^{2} \pi^{2} D t}{4 L^{2}}} \operatorname{Exp}[U] \times \frac{-4 L^{2}}{(2 n+1)^{2} \pi^{2} D} d U
$$

Rearranging,

$$
\begin{aligned}
& M_{t}=\frac{4 D\left(C_{1}-C_{0}\right)\left(-4 L^{2}\right)}{L(2 n+1)^{2} \pi^{2} D} \int_{0}^{-\frac{(2 n+1)^{2} \pi^{2} D t}{4 L^{2}}} \operatorname{Exp}[U] d U \\
& M_{t}=\left.\frac{4\left(C_{1}-C_{0}\right)(-4) L}{(2 n+1)^{2} \pi^{2}}(\operatorname{Exp}(U))\right|_{0} ^{\frac{(2 n+1)^{2} \pi^{2} D t}{4 L^{2}}} \\
& M_{t}=-\frac{16\left(C_{1}-C_{0}\right) L}{(2 n+1)^{2} \pi^{2}}\left[\operatorname{Exp}\left(-\frac{(2 n+1)^{2} \pi^{2} D t}{4 L^{2}}\right)-1\right] \\
& M_{t}=\frac{16\left(C_{1}-C_{0}\right) L}{\pi^{2}} \sum_{n=0}^{\infty} \frac{1}{(2 n+1)^{2}}\left[1-\operatorname{Exp}\left(-\frac{(2 n+1)^{2} \pi^{2} D t}{4 L^{2}}\right)\right]
\end{aligned}
$$

For the infinite time the amount of substance entered or left the sheet can be defined as [21],

$$
M_{\infty}=2\left|C_{1}-C_{0}\right| L
$$

And the total amount of diffusing substance which has entered or left the sheet of thickness $2 L$ (from $-L$ to $L$ ) at time $t, M_{t}$ is expressed as a fraction of the corresponding quantity after infinite time $M_{\infty}$ as,

$$
\frac{M_{t}}{M_{\infty}}
$$

Then substituting Eqns. (39) and (40) into Eqn. (41) we get,

$$
\frac{M_{t}}{M_{\infty}}=\frac{\frac{16\left(C_{1}-C_{0}\right) L}{\pi^{2}} \sum_{n=0}^{\infty} \frac{1}{(2 n+1)^{2}}\left[1-\operatorname{Exp}\left(-\frac{(2 n+1)^{2} \pi^{2} D t}{4 L^{2}}\right)\right]}{2\left|C_{1}-C_{0}\right| L}
$$




$$
\begin{aligned}
& \frac{M_{t}}{M_{\infty}}=\frac{8}{\pi^{2}} \sum_{n=0}^{\infty} \frac{1}{(2 n+1)^{2}}\left[1-\operatorname{Exp}\left(-\frac{(2 n+1)^{2} \pi^{2} D t}{4 L^{2}}\right)\right] \\
& \frac{M_{t}}{M_{\infty}}=\frac{8}{\pi^{2}}\left[\sum_{n=0}^{\infty} \frac{1}{(2 n+1)^{2}}-\sum_{n=0}^{\infty} \frac{1}{(2 n+1)^{2}} \operatorname{Exp}\left[-\frac{(2 n+1)^{2} \pi^{2} D t}{4 L^{2}}\right]\right]
\end{aligned}
$$

From the series rule,

$$
\sum_{n=0}^{\infty} \frac{1}{(2 n+1)^{2}}=\frac{\pi^{2}}{8}
$$

Then,

$$
\frac{M_{t}}{M_{\infty}}=\frac{8}{\pi^{2}}\left[\frac{\pi^{2}}{8}-\sum_{n=0}^{\infty} \frac{1}{(2 n+1)^{2}} \operatorname{Exp}\left[-\frac{(2 n+1)^{2} \pi^{2} D t}{4 L^{2}}\right]\right]
$$

Rearranging

$$
\begin{aligned}
& \frac{M_{t}}{M_{\infty}}=\frac{8 \pi^{2}}{\pi^{2} 8}-\frac{8}{\pi^{2}} \sum_{n=0}^{\infty} \frac{1}{(2 n+1)^{2}} \operatorname{Exp}\left[-\frac{(2 n+1)^{2} \pi^{2} D t}{4 L^{2}}\right] \\
& \frac{M_{t}}{M_{\infty}}=1-\sum_{n=0}^{\infty} \frac{8}{(2 n+1)^{2} \pi^{2}} \operatorname{Exp}\left[-\frac{(2 n+1)^{2} \pi^{2} D t}{4 L^{2}}\right]
\end{aligned}
$$

For the alternating solution Laplace transformation will result [21]

$$
\frac{M_{t}}{M_{\infty}}=4\left[\frac{D t}{l^{2}}\right]^{1 / 2}\left[\frac{1}{\pi^{\frac{1}{2}}}+2 \sum_{n=1}^{\infty}(-1)^{n} \operatorname{ierfc} \frac{n l}{2 \sqrt{D t}}\right]
$$

For the small times short time behavior sum part, has no effect to the summation after first few terms. So $\sum \approx 1 / \sqrt{\pi}$.

$$
\frac{M_{t}}{M_{\infty}}=4\left[\frac{D t}{\pi l^{2}}\right]^{1 / 2}
$$

In Eqn. (44), constants $D, \pi$, and $l$ can be collected under a new constant $k$.

$$
\frac{M_{t}}{M_{\infty}}=k t^{1 / 2}
$$

For a second limiting case, according to kinetics of zero order, the drug release rate is independent of time. Such situation is described by a general equation of the form 


$$
\frac{M_{t}}{M_{\infty}}=k t
$$

Most of the release falls between the Eqns. (45) and (46). So, both equations could be summed.

$$
\frac{M_{t}}{M_{\infty}}=k_{1} \sqrt{t}+k_{2} t
$$

Eqn. (47) can be generalized as,

$$
\frac{M_{t}}{M_{\infty}}=k t^{n}
$$

Eqn. (48) is the Peppas model which is very similar to the Higuchi model. One should focus on the exponential part of the time which is not constant. Fitting model to the experimental data with variable data will be slightly different from the routine process. Which will be discussed in further chapters.

\subsection{Hixon Crowell Model}

Hixon Crowell model is another popular model in the field [22]. It is not as popular as Higuchi and Peppas model but, considering its derivation, it is worth to mention. In Hixon Crowell model the basic concept can be specified as,

$$
\underbrace{\frac{d C(t)}{d t}}_{\begin{array}{c}
\text { rate of } \\
\text { concentration } \\
\text { change }
\end{array}}=k\left(C_{s}-C(t)\right)
$$

Where $C(t)$ is the concentration depends on time, $k$ is constant, $C_{s}$ is the concentration of the saturated solution. Rate of change of weight described as,

$$
\frac{d w}{d t}=k_{2} S\left(C_{s}-C\right)
$$

Where $w, k_{2}$ and $S$ are wieght, constant and surface of substance at time $t$ respectively. If weight of the substance dissolved at timet,

$$
w_{0}-w
$$

Then the mass in a specific volume is,

$$
\frac{\left(w_{0}-w\right)}{V}=C
$$


Where $V$ is volume, and,

$$
\frac{w_{s}}{V}=C_{s}
$$

If Eqns. (52) and (53) substituted into Eqn. (50)

$$
\frac{d w}{d t}=-k_{2} S\left(\frac{w_{s}}{V}-\frac{w_{0}-w}{V}\right) \rightarrow \quad V\left(\frac{d w}{d t}\right)=-k_{2} S\left(w_{s}-w_{0}+w\right)
$$

Surface varies $2 / 3$ power of its volume.

$$
S=V^{2 / 3} \quad, \quad V=\frac{w}{d} \quad \rightarrow \quad S=\underbrace{\frac{1}{d^{2 / 3}}}_{k} w^{2 / 3}
$$

Where $d$ is density and $k$ is constant which represents density. Then $S$ is substituded into Eqn. (54).

$$
V\left(\frac{d w}{d t}\right)=-k_{2} k w^{2 / 3}\left(w_{s}-w_{0}+w\right)
$$

Collecting constants $k_{2}$ and $k$ into a new constant as $k_{1}$, defining $w_{s}-w_{0}=g$ and rearranging Eqn. (56)

$$
\begin{aligned}
& V\left(\frac{d w}{d t}\right)=-k_{1} w^{2 / 3}(g+w) \rightarrow V\left(\frac{d w}{d t}\right)=-k_{1}\left(g w^{2 / 3}+w^{5 / 3}\right) \\
& V \times \frac{1}{\left(g w^{2 / 3}+w^{5 / 3}\right)} d w=-k_{1} d t
\end{aligned}
$$

Integrating both parts

$$
\begin{aligned}
& V \int \frac{1}{\left(g w^{2 / 3}+w^{5 / 3}\right)} d w=-k_{1} \int d t \\
& g^{1 / 3}=a \quad, \quad w_{0}^{1 / 3}=b \quad, \quad w^{1 / 3}=x \quad, \quad w=x^{3}, \quad d w=3 x^{2} d x \\
& V \int \frac{3 x^{2}}{\left(a^{3} x^{2}+x^{5}\right)} d x=\frac{V\left(2 \sqrt{3} \tan ^{-1}\left[\frac{(-a+2 x)}{\sqrt{3} a}\right]+2 \ln [a+x]-\ln \left[a^{2}-a x+x^{2}\right]\right)}{2 a^{2}}
\end{aligned}
$$




$$
a=g^{1 / 3} \quad \rightarrow \quad g=\left(w_{s}-w_{0}\right) \quad, \quad x=w^{1 / 3}
$$

While concentration change of a substance is negligible. So $C$ is constant then $C_{s}-C$ is constant. The rate is proportional to the surface alone, which is,

$$
\frac{d w}{d t}=-k_{3} w^{2 / 3}
$$

Integrating both parts,

$$
\int_{0}^{t} \frac{1}{w^{2 / 3}} d w=-k_{3} \int d t \quad \rightarrow \quad w_{0}^{1 / 3}-w^{1 / 3}=k_{4} t
$$

Eqn. (58) could be considered as Hixon Crowell model [22].

\section{Experimental Analysis}

For the experimental analysis part, public data table "Cumulative \% drug release data of all the formulations" [23] is used from the work of Madhavi [24], under the creative commons license by, CC BY-NC-SA 3.0 [25]. The work corresponds the dissolution enhancement of efavirenz by solid dispersion and PEGylation techniques. In related work, PEGylated compound 2 is released with time by $(5,10,15,20,25,30,45,60,80,100)$ minutes with $(17,22,32,34,39,42,48,50,54,57)$ percentage which is sufficient for fitting and testing the models.

For the simplicity and linearity of the Zero Order model as seen in Eqn. (4), the fit of it does not require modification of axes to get a suitable representation. All the data collected in a suitable matrix and fitted to the function. In this article the Wolfram Mathematica 10 software is used to perform fitting, graphing and strength test.

For the Zero Order model it could be observed that fit does not look good enough because of the fit function is missing most of the data points. But when it is coming to compare many models visual comments might start debate. To test the strength of the models quantitatively it is chosen Watson's U Square Method [26] because of the convenience of the data set. This test yields two results. One statistic and other P-Value. For the perfect fit statistic value should be equal to zero and P-Value should be equal to one. Then it could be commented on the strength test results comparison. Which model is strong or weak and which model is better? For the Figure 3 fit results, strength test yield statistic 0.0605 and P-Value 0.615658.

Fitting the First Order model is slightly different from the Zero Order model, because of the model functions logarithmic nature as seen in Eqn. (8). For this kind of equation, it is not possible to draw a graph as drug release versus time, because the function represents logarithm of the remaining drug. To overcome this axes problem, data matrix should be converted to the suitable form by first, converting release data into remaining data. Second, convert it into a logarithmic value. Then, fitting the function will be possible. 


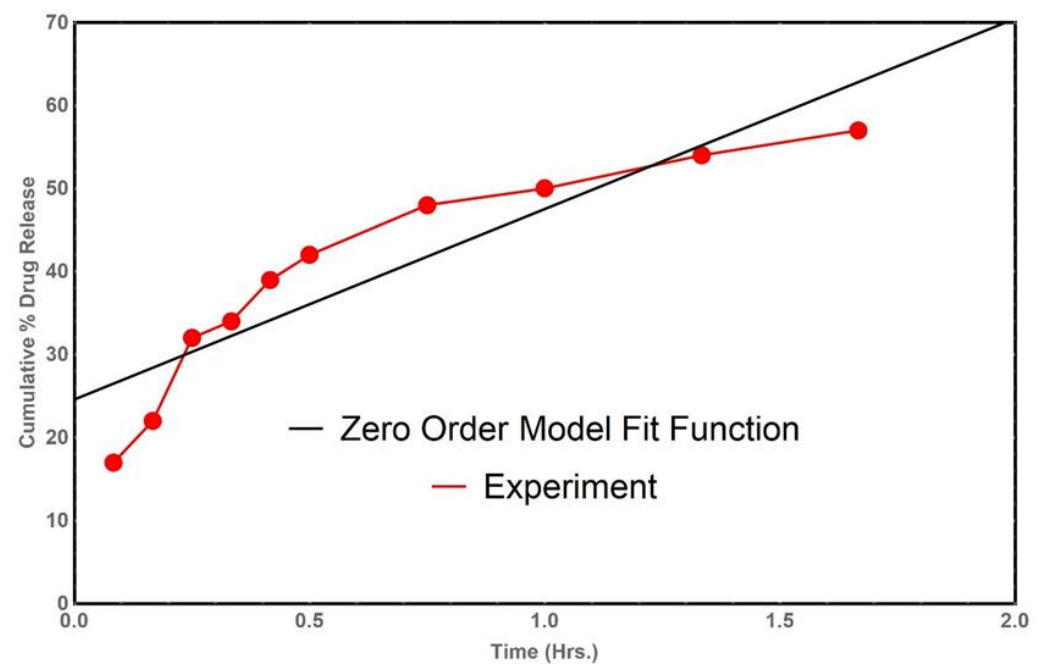

Fig. 3 Fit of Zero Order model function to the drug release data.

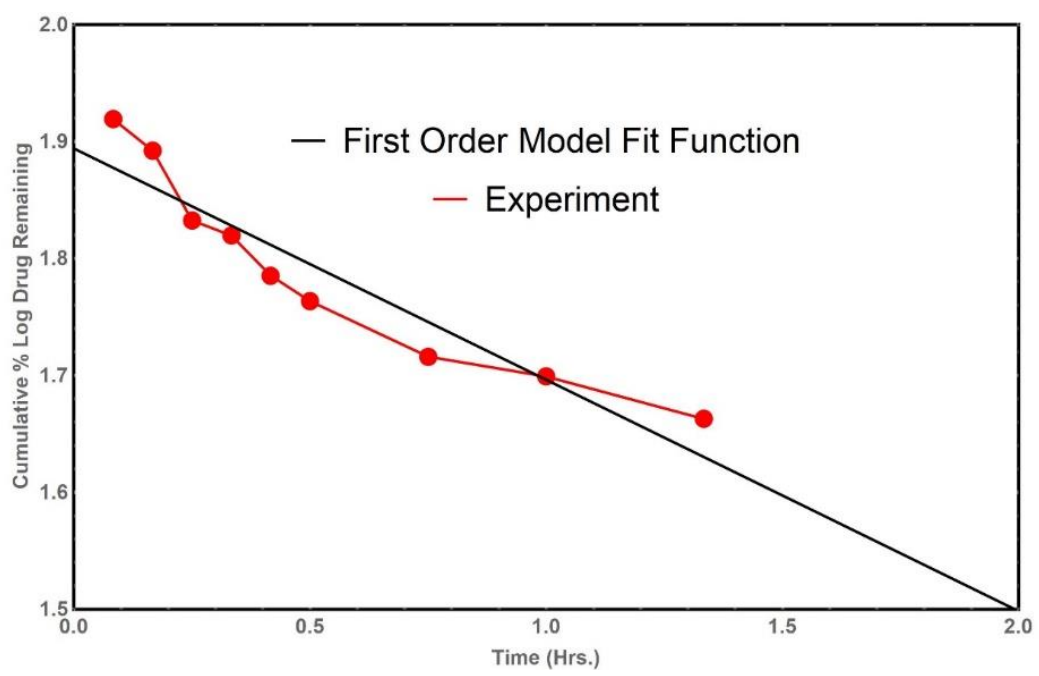

Fig. 4 Fit of First Order model function to the drug release data.

As seen from the Figure 4, vertical axes is different from the Figure 3. And function behaves linear with negative slope thanks to nature of the function. Strength test results as statistic 0.403509 and P-Value 0.000565739.

As it seen, every model needs specific data set arrangements for fitting. For Higuchi model, drug release remains same but time varies with the power of $1 / 2$ so it will not appropriate to use same horizontal axes with Zero and First Order models. Time part of the data matrix also should be converted into suitable form by adding the power of $1 / 2$ to it as, 


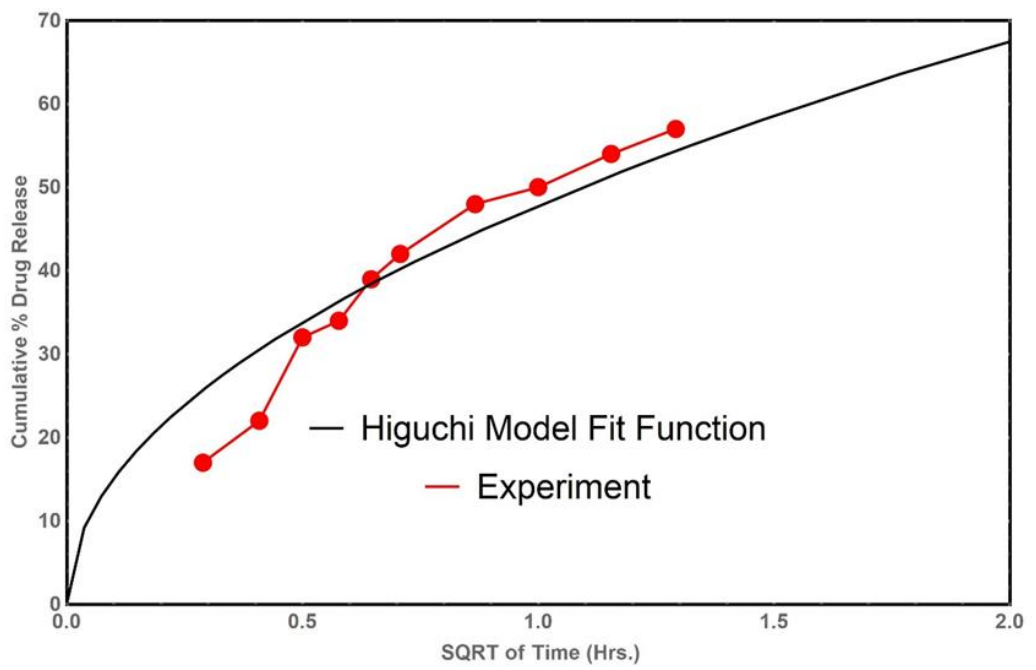

Fig. 5 Fit of Higuchi model function to the drug release data.

As it seen from the Figure 5vertical axis is similar with the Zero Order model, but horizontal axis is converted into Higuchi form. Higuchi Eqn. (13) predicts fast diffusion for short term first, then release starts to get slower with time thanks to the $1 / 2$ power of time. That makes sense for most diffusion-based release, and that is why it stands one of the strongest and popular model of all time. For the fit in Figure 5, Higuchi model strength test results 0.0445 for statistic and 0.808438 for P-Value.

In Peppas model time varies with the power of a parameter $n$ as seen in Eqn. (48). In this case it will not be possible to generate graph for an unknown axis. But fitting procedure still will generate the parameters. So, data matrix could be converted into Peppas form with the unknown parameter $n$ than fitting is applied. In that way software will generate the most possible parameters for both $k$ and $n$ values which makes it possiple to visualize.

As seen from the Figure 6 fit function gets close to the dataset and horizontal axes is converted into the Peppas form with the generated parameter. Strength test of the Peppas model yields, statistic 0.033 and P-Value 0.933232.

For another popular model Hixon Crowell model to arranging data matrix is follows the same procedure. As seen in Eqn. (58) function represents difference between cube roots of the initial amount and remaining amount. Applying this difference into the data matrix, it easily could be getting the vertical axes. 


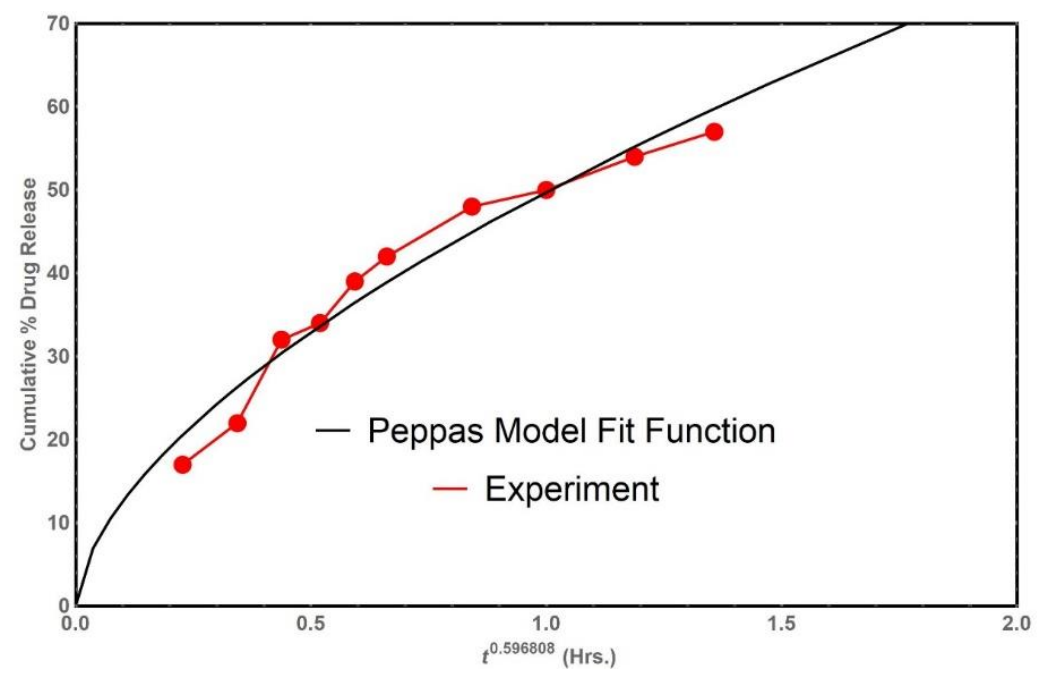

Fig. 6 Fit of Peppas model function to the drug release data.

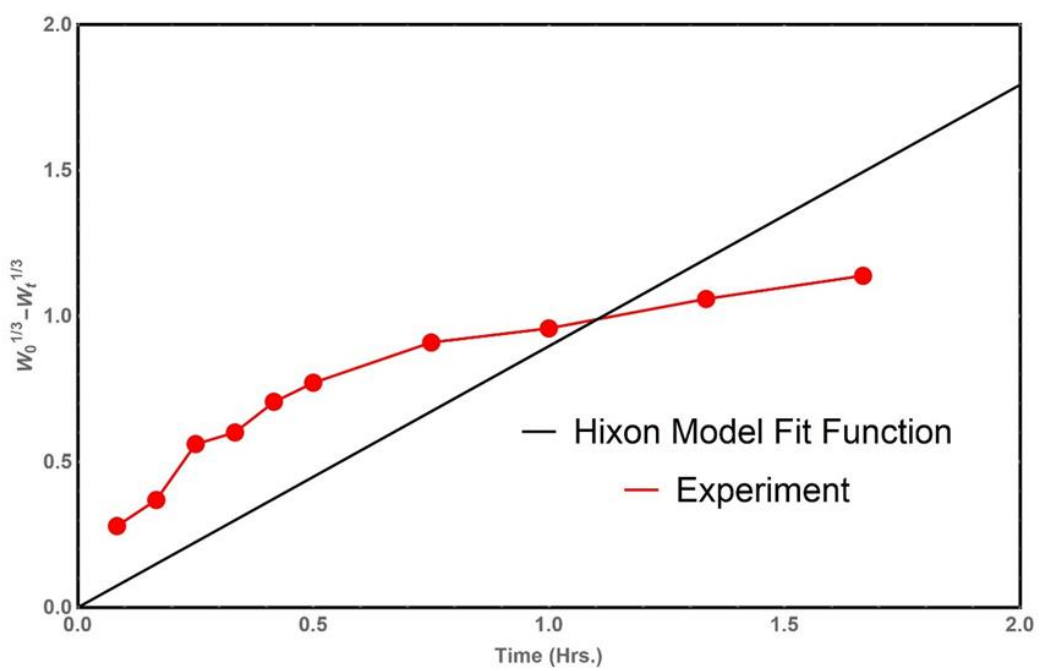

Fig. 7 Fit of Hixon Crowell model function to the drug release data.

It could easily be observed that the vertical axis has transformed the model suitable form from the Figure 7. Strength test of Hixon Crowell model results the statistic 0.425 and PValue 0.00039154 which is far from a good fit because the model function is stacked to the origin. 
Table 1 Model fit parameters.

\begin{tabular}{cccc}
\hline \multirow{2}{*}{ Models } & \multicolumn{3}{c}{ Parameters } \\
\cline { 2 - 4 } & $\mathrm{C}$ & $\mathrm{K}$ & $\mathrm{n}$ \\
\hline Zero Order Model & 24.5908 & 22.9373 & - \\
First Order Model & 78.3375 & 0.4555 & - \\
Higuchi Model & - & 47.8268 & - \\
Peppas Model & - & 49.8155 & 0.5968 \\
Hixon Crowell & - & 0.8965 & - \\
Model & - & & \\
\hline
\end{tabular}

Table 2 Comparison of the strength of the fits.

\begin{tabular}{ccc}
\hline Models & Statistic & P-Value \\
\hline Zero Order Model & 0.060500 & 0.615658 \\
First Order Model & 0.403509 & 0.000565 \\
Higuchi Model & 0.044500 & 0.808438 \\
Peppas Model & 0.033000 & 0.933232 \\
Hixon Crowell & 0.425000 & 0.000391 \\
Model & &
\end{tabular}

As seen from the

Table 2, Higuchi and Peppas models are able to generate strong fits to the drug release data.

Finally it is seen from the experimental analysis, Zero Order model and First Order model can be applied for the $50 \%$ of the drug release approximately for the related data. However Higuchi and Peppas model can generate fit function up to $60 \%$ of drug release which is end of the release data. But the curvature of the fit functions foreseen that, the function is able to produce higher percentage representation. Also Hixon model is not as good as Higuchi and Peppas model because of its origin dependent nature. This behavior of the models is obviously occurred by the experimental details. In the related data set, drug is released up to $57 \%$. In many experiments sink conditions is not satisfied and due to the saturation drug release is slowed, or in some scenarios there are some drug carriers that prevents the fast release. All the related parameters should be added into the models carefully if needed

\section{Conclusions}

In the DD field, there is no comprehensive resource describing the derivation of mathematical models explicitly. Existing works either focus on single model or jump into direct result of model functions. The purpose of this review is showing explicit derivation of the most popular models and collecting them under one single paper. At the same time, the problems encountered during calculation and fitting are also discussed in detail. These problems are not clarified in the literature.

The first of the two major problems is choosing the correct expression for release when applying boundary conditions (Eqn. (27)). There is no guide in the literature to the selecting boundary conditions correctly and to obtain parameters. This study might fill that gap by the explicit applications of the boundaries without non-dimensionalization. Second problem is the representation of the fitting functions in proper axes. In most of the studies, fitting is represented by build-in ready computer software and the obtained results are shown directly. Almost every specialist uses that way because it is faster and easier. But 
there is still no guide to align the axes for the relevant model. This review also overcome that problem (Figure 4-Figure 7))

As a result, in this study it is focused on guiding the new researchers who interests to understand drug delivery mechanism by collecting all necessary subjects under one single source. Derivation, calculation, application and testing of the models have been explained carefully which might help scientists to take their works further in related area. Also, it is underlined the importance of the models that give predictions to the scientists for preventing waste of time and money for their experiments. In the future it will be focused on the relation between the reaction rate constant and activation energy by Arrhenius equation, which leads to the calculations to the thermodynamic studies. Thus, it is planned to investigate whether there is a relationship between entropy and enthalpy and the activation energy of the reaction.

\section{Conflict of interest}

The authors declare that they have no known competing financial interests or personal relationships that could have appeared to influence the work reported in this paper.

\section{Acknowledgement}

The authors acknowledge that this study is supported by BAP research project in the University of Ondokuz Mayıs (Project Number: PYO.FEN.1901.18.008).

\section{References}

[1] Siepmann J, Peppas NA. Modeling of drug release from delivery systems based on hydroxypropyl methylcellulose (HPMC). Advanced Drug Delivery Reviews, 2001; 48:23 139-157. https://doi.org/10.1016/S0169-409X(01)00112-0

[2] Siepmann J, Gopferich A. Mathematical modeling of bioerodible, polymeric drug delivery systems. Advanced Drug Delivery Reviews, 2001; 48:2-3 229-247. https://doi.org/10.1016/S0169-409X(01)00116-8

[3] Arifin DY, Lee LY, Wang CH. Mathematical modeling and simulation of drug release from microspheres: Implications to drug delivery systems. Advanced Drug Delivery Reviews, 2006; 58:12-13 1274-1325. https://doi.org/10.1016/j.addr.2006.09.007

[4] Lin CC, Metters AT. Hydrogels in controlled release formulations: Network design and mathematical modeling. Advanced Drug Delivery Reviews, 2006; 58:12-13 1379-1408. https://doi.org/10.1016/i.addr.2006.09.004

[5] Brochmann-Hanssen E, Higuchi T. Pharmaceutical analysis, Interscience Publishers, New York,, 1961.

[6] Grassi M, Voinovich D, Moneghini M, Franceschinis E, Perissutti B, Filipovic-Grcic J. Preparation and evaluation of a melt pelletised paracetamol/stearic acid sustained release delivery system. Journal of Controlled Release, 2003; 88:3 381-391. https://doi.org/10.1016/S0168-3659(03)00011-7

[7] Grassi M, Zema L, Sangalli ME, Maroni A, Giordano F, Gazzaniga A. Modeling of drug release from partially coated matrices made of a high viscosity HPMC. International Journal of Pharmaceutics, 2004; 276:1-2 107-114. https://doi.org/10.1016/j.ijpharm.2004.02.016

[8] Ju RT, Nixon PR, Patel MV. Drug-Release from Hydrophilic Matrices .1. New Scaling Laws for Predicting Polymer and Drug-Release Based on the Polymer Disentanglement Concentration and the Diffusion Layer. Journal of Pharmaceutical Sciences, 1995; 84:12 1455-1463. https://doi.org/10.1002/jps.2600841213

[9] Ju RT, Nixon PR, Patel MV, Tong DM. Drug-Release from Hydrophilic Matrices .2. A Mathematical-Model Based on the Polymer Disentanglement Concentration and the 
Diffusion Layer. Journal of Pharmaceutical Sciences, 1995; 84:12 1464-1477. https://doi.org/10.1002/jps.2600841214

[10] Ju RT, Nixon PR, Patel MV. Diffusion coefficients of polymer chains in the diffusion layer adjacent to a swollen hydrophilic matrix. Journal of Pharmaceutical Sciences, 1997; 86:11 1293-1298. https://doi.org/10.1021/js970053n

[11] Siepmann J, Podual K, Sriwongjanya M, Peppas NA, Bodmeier R. A new model describing the swelling and drug release kinetics from hydroxypropyl methylcellulose tablets. Journal of Pharmaceutical Sciences, 1999; 88:1 65-72. https://doi.org/10.1021/js9802291

[12] Siepmann J, Peppas NA. Hydrophilic matrices for controlled drug delivery: an improved mathematical model to predict the resulting drug release kinetics (the "sequential layer" model). Pharm Res, 2000; 17:10 1290-8.

[13] Siepmann J, Kranz H, Peppas NA, Bodmeier R. Calculation of the required size and shape of hydroxypropyl methylcellulose matrices to achieve desired drug release profiles. International Journal of Pharmaceutics, 2000; 201:2 151-64. https://doi.org/10.1016/S0378-5173(00)00390-2

[14] Harland RS, Gazzaniga A, Sangalli ME, Colombo P, Peppas NA. Drug/polymer matrix swelling and dissolution. Pharm Res, 1988; 5:8 488-94. https://doi.org/10.1023/A:1015913207052

[15] Narasimhan B, Peppas NA. Molecular analysis of drug delivery systems controlled by dissolution of the polymer carrier. J Pharm Sci, 1997; 86:3 297-304. https://doi.org/10.1021/is960372z

[16] Kalyanasundaram S, Calhoun VD, Leong KW. A finite element model for predicting the distribution of drugs delivered intracranially to the brain. Am J Physiol, 1997; 273:5 R1810-21. https://doi.org/10.1152/ajpregu.1997.273.5.R1810

[17] Lankelma J, Fernández Luque R, Dekker H, Schinkel W, Pinedo HM. A mathematical model of drug transport in human breast cancer. Microvasc Res, 2000; 59:1 149-61. https://doi.org/10.1006/mvre.1999.2218

[18] Fick A. On Liquid Diffusion (Reprinted from the London, Edinburgh, and Dublin Philosophical Magazine and Journal of Science, Vol 10, Pg 30,1855). Journal of Membrane $\quad$ Science, $\quad 1995 ; \quad 100: 1 \quad 33-38$. https://doi.org/10.1080/14786445508641925

[19] Crank J. The mathematics of diffusion / by J. Crank, Clarendon Press, Oxford [England], 1975.

[20] Caccavo D. An overview on the mathematical modeling of hydrogels' behavior for drug delivery systems. International Journal of Pharmaceutics, 2019; 560:175-190. https://doi.org/10.1016/j.ijpharm.2019.01.076

[21] Ritger PL, Peppas NA. Transport of Penetrants in the Macromolecular Structure of Coals .7. Transport in Thin Coal Sections. Fuel, 1987; 66:10 1379-1388. https://doi.org/10.1016/0016-2361(87)90185-2

[22] Hixson AW, Crowell JH. Dependence of Reaction Velocity upon Surface and Agiation. Industrial and Engineering Chemistry, 1931; 923-931. https://doi.org/10.1021/ie50260a018

[23] Madhavi B. Cumulative \% drug release data of all the formulations, 2011.

[24] Madhavi BB, Kusum B, Chatanya ChK, Madhu MN, Harsha VS, Banji D. Dissolution enhancement of efavirenz by solid dispersion and PEGylation techniques. Int J Pharm Investig, 2011; 1:1 29-34. https://doi.org/10.4103/2230-973X.76726

[25] Creative Commons Licence 3.0. https://creativecommons.org/licenses/by-nc$\mathrm{sa} / 3.0 /$.

[26] Batschelet E. United States. Office of Naval Research., Statistical methods for the analysis of problems in animal orientation and certain biological rhythms, American Institute of Biological Sciences, Washington, 1965. 